\title{
Del proyecto a la obra: la producción de saber en el campo de las prácticas estético-artísticas contemporáneas
}

\section{From project to artwork: the production of knowledge in the field of contemporary esthetic-artistic practices}

TIPO DE TRABAJO: Comunicación.

PALABRAS CLAVE

Proyecto, obra, prácticas estético-artísticas, investigación-creación.

KEY WORDS

Project, art work, esthetic-artistic practices, research-creation processes.

RESUMEN:

Las rupturas que ha experimentado el campo de las artes visuales y las humanidades desde la última década del siglo XX, han incidido de manera directa en los modos como se produce obra en estos ámbitos. Por un lado, los artistas y creadores se han visto impulsados a incorporar a sus prácticas, metodologías provenientes de las ciencias sociales y de otras disciplinas, para abordar las realidades que los comprometen. Por otro lado, los investigadores sociales han encontrado en los procesos expresivos y en las representaciones simbólicas, dispositivos fundamentales para problematizar sus objetos de estudio y dar cuenta de realidades que escapan a la mera textualidad. En este intercambio de experiencias, saberes y métodos, ha ido tomando cuerpo la noción de investigación-creación, desde apuestas que logran legitimidad, bien en espacios de formación o bien en escenarios propiamente creativos, en los que se convoca la inter y la transdisciplinariedad.

La reflexión que proponemos, retoma los aportes y planteamientos conceptuales de autores como Borgdorf, Rancière, Sennet, Nancy, entre otros, además de la propia experiencia derivada de la Maestría en Estética y Creación de la UTP con el fin de problematizar los intercambios que tienen lugar en los procesos de investigación-creación, a partir de la actualización de dos nociones ancladas a la tradición, tanto de la investigación formal, como de la creación artística: las nociones de proyecto y obra. En la misma perspectiva, problematiza dos ámbitos de experimentación y creación, afianzados en los procesos de producción de sentido en estos campos: el taller y el laboratorio.

Los planteamientos desarrollados se derivan del proyecto: "Investigación-creación: Tensiones y apropiaciones en la proyección de las prácticas estético artísticas contemporáneas", desarrollado por los profesores Aura Margarita Calle y Felipe Martínez, del Departamento de Humanidades de la Universidad Tecnológica de Pereira, Colombia.

\section{ABSTRACT:}

The ruptures experienced in the field of visual arts and humanities since the last decade of the twentieth century have directly influenced the ways in which artwork is produced in these areas. On the one hand, artists and creators have been encouraged to incorporate into their practices, methodologies from social sciences and other disciplines, to address the realities that determine them. On the other hand, social researchers have found in expressive processes and in symbolic representations, fundamental devices 
to problematize their objects of study and regard for realities that escape single textuality. In this exchange of experiences, knowledge and methods, the notion of Research-creation has taken shape, from leads that achieve legitimacy, either in training spaces or in properly creative scenarios, in which inter and transdisciplinarity are convened.

The reflection that we propose, takes up the contributions and conceptual approaches of authors such as Borgdorf, Rancière, Sennet, Nancy, among others, in addition to the own experience derived from the Master in Esthetics and Creation from UTP in order to problematize the exchanges that take place in the research-creation processes, From the updating of two notions anchored to the tradition, both formal research and artistic creation: the notions of project and artwork. In the same perspective, it problematizes two areas of experimentation and creation, entrenched in the processes of production of meaning in these fields: the workshop and the laboratory.

The approaches developed are derived from the project: "Research-creation: Tensions and appropriations in the projection of contemporary esthetic and artistic practices", developed by professors Aura Margarita Calle y Felipe Martínez from the Department of Humanities of the Universidad Tecnológica de Pereira, Colombia.

\section{INTRODUCCIÓN}

En el ámbito de las prácticas estético-artísticas asistimos a una redefinición de los modos de crear y a un impulso por asociar esta acción con la práctica investigativa. Esta situación es sintomática y responde a factores tanto de orden estético y cultural, como a exigencias de orden académico e institucional. Sólo al finalizar los años 90 del siglo XX, y de manera más contundente, en lo que va corrido del presente siglo, se empieza a manifestar el interés por reflexionar el sobre la posibilidad de vincular la creación artística con la investigación. La idea de una producción plástica afianzada exclusivamente en la labor práctica, es decir, en el proceso configurador, ha respondido al paradigma creativo del arte autónomo, heredado de la tradición, que se anidó en las facultades de artes sin que se cuestionara su validez estética y social. Hoy, sin embargo, estos modos de crear, están bajo sospecha, pues además de capacidad para crear acontecimientos sensibles, la academia reclama de los creadores el despliegue de nuevas competencias cognitivas, para movilizar pensamiento y animar la reflexividad en torno a los procesos que desarrollan. No de otra manera se entiende que estemos asistiendo a la construcción de un nuevo marco de comprensivo para las prácticas artísticas y creativas en el que aparecen comprometidas nociones ancladas al imaginario de la creación visual, como las de obra y taller, ahora desplazadas o equiparadas a las de proyecto y laboratorio.

\section{METOdOLOGÍA}

Hasta bien avanzado el siglo XX, el abordaje de los problemas vinculados al campo de la estética y la creación se desplazaron dentro de marcos interpretativos y procedimentales, que optaron por privilegiar unas epistemes y unas estructuras conformativas en detrimento de otras. En nuestro momento actual tales procesos han sido objeto de aperturas y replanteamientos que han colocado la investigación-creación en un campo de cruces e intercambios disciplinares que buscan validar nuevos modos de identificar significados y construir posturas alrededor de los fenómenos del arte, la estética y la cultura. En coherencia con estas dinámicas, nos ocuparemos de visibilizar la concreción de tales desplazamientos con miras a despejar posibles rutas para abordar los procesos de investigación-creación en una época en la que las prácticas estético-artísticas aparecen imbricadas en un campo complejo de relaciones conformado por saberes, tradiciones, instituciones y efectos pragmáticos, que hacen de tales prácticas una auténtica sedimentación de lo que podemos reconocer como procesos culturales.

Los presupuestos que articulan esta propuesta se derivan de la experiencia de enfrentar, desde hace más de una década, la tarea de intentar comprender las lógicas de producción de las artes plásticas, sobre todo en una época en la que los pluralismos expresivos y los intercambios disciplinares tiende a relativizar los procesos que conciernen a la pregunta por el lugar de la investigación en la creación artística, al tiempo que las instituciones arte y la academia usan y abusan del sentido de la relación investigar-crear en convocatorias, programas de formación y escenarios de legitimación del trabajo creativo, esquivando el debate sobre las reglas de juego para visibilizar los proyectos y la producción artística que promueven. 


\section{DESARROLLO}

Dos procesos nos permiten ubicar el nuevo marco comprensivo en el que se instalan las formas de producción y mediación derivadas de las prácticas artísticas y creativas y, por ende, las relaciones que tales prácticas promueven en relación con los discursos y formas de saber que se proyectan en la construcción de conocimiento a partir del siglo XX: la expansión del ámbito de dominio de la estética y la mutación del campo de las artes.

\section{La expansión del ámbito de dominio de la estética}

La idea de una estética no restringida es relativamente reciente. Cuando hablamos de estéticas expandidas o ampliadas hacemos referencia al giro que comprometió su sentido con los fenómenos que tienen lugar en el mundo social y cultural, lo que, de manera correlativa posibilitó una nueva mirada sobre el arte y la manera como este se acercaba a los fenómenos que abordaba: “...ninguna cosa es estética en sí misma, ni siquiera las obras de arte o las cosas bellas. La estesis está en las relaciones que propician los sujetos, no en las cosas.", advierte Katya Mandoki $(2008$, p.50)

En otra vía, Jacques Rancière (2013), precisa como este giro ha implicado la revaloración de la estética dentro de un régimen específico de identificación y pensamiento de las artes que complejiza tanto "las maneras de hacer", como "las formas de visibilidad de esas maneras de hacer y los modos de pensabilidad de sus relaciones". Es decir, implica tanto el régimen de funcionamiento del arte, como sus matrices discursivas, las formas de identificación de lo propio del arte (y, en consecuencia, lo que no es propio de las prácticas artísticas) y la redistribución de las relaciones entre las formas de la experiencia sensible. (p. 24). Este giro ha sido tematizado en una abundante literatura, que se ha encargado de focalizar el modo como la estética y lo estético atraviesa nuestras diferentes maneras de estar en el mundo, cobijando al arte, por su puesto, pero sin que este sea su único detonante.

\section{Mutaciones del campo de las artes}

Un segundo factor decisivo en la redefinición de las visualidades contemporáneas se puede observar en la propia transformación que ha sufrido el campo del arte, sobre todo a lo largo del siglo XX y lo que va corrido del XXI. En este lapso de tiempo la producción artística dejó de estar afianzada en un programa específico, centrado en comportamientos y valores con cierta aspiración de objetividad, que limitaban la calidad de las obras a la solución de problemas técnicos y formales, mientras que el aparataje institucional dio cabida a prácticas de competencia y a presupuestos críticos más plurales y flexibles. Tales desplazamientos nos han permitido tematizar, por lo menos, tres momentos, a saber:

- Primero, un "antes" circunscrito al arte clásico, en el cual los artistas se dedicaban a "la representación del mundo [...] tal como se les presentaba o hubieran presentado al ojo" (Danto, 1999, p.28), construyendo una relación de "adecuación" o conformidad entre lo que se representa y lo representado, que se circunscribía a la maestría del creador y se limitaba al despliegue técnico del oficio.

- Segundo, un "después" en el que se configura lo que reconocemos como arte formal, se proclama la autonomía del arte, la conquista de lo inédito como fin y el ensimismamiento del artista como mecanismo para el apropiado seguimiento de los preceptos estatuidos por la disciplina. De esa voluntad de aislamiento del artista surge la noción del "arte por el arte", como referente de identidad de un arte autónomo, en el que según Greenberg (1979, p. 14), la creación tenía validez en sí misma, "de la misma manera que la naturaleza es válida en sí misma"

- Y tercero, el "fin del arte" que interpreta el giro operado por los propios artistas de la vanguardia, quienes, agotados en el dogmatismo asfixiante del sistema formal, lo fueron minando hasta detonarlo.

En el extravío de sus límites los artistas y sus creaciones pierden el sostén de la autocomprensión que era garante de la existencia del arte como lugar de la verdad y como experiencia de comunión con los hombres. Como consecuencia de esto se instala la necesidad de un nuevo êthos cultural y expresivo, capaz de condensar y exteriorizar críticamente el lugar que empiezan a redefinir los sistemas de formalización y de interpretación de los lenguajes artísticos, incluida la desestructuración de los sistemas nucleares de la representación.

Es precisamente en este marco de referencia, que la relación entre crear e investigar adquiere un matiz renovado, que obliga a pensar tales nociones como complementarias y en una condición de horizontalidad y retribución mutua. Esta es la vía que asume Borgdorff (2012) al ocuparse de tematizar la creación y la investigación en el contexto de las artes contemporáneas. Para este autor, existen tres modos de abordar la creación-investigación en el campo de las artes: Investigación sobre las artes, investigación para las artes e investigación en las artes. Por investigación sobre las artes, podríamos comprender las apuestas de carácter reflexivo, las cuales permiten tematizar aspectos relacionados con los procesos creativos desde una perspectiva interpretativa y/o histórica, mientras que la Investigación para las artes, puede describirse como un tipo de estudio al servicio de la práctica artística, para configurar las herramientas, el conocimiento técnico sobre los materiales, necesario para el proceso creativo o el producto artístico final. Por último, 
la investigación en las artes, sintetiza una perspectiva en la cual no es posible trazar distancia entre la investigación y la práctica artística, pues es justamente en la relación estrecha que traman estas dos acciones donde tiene lugar la obra.

Ahora bien, en aras de apuntar hacia posibilidades de validación tanto en términos académicos como institucionales, los procesos de investigación-creación o de investigación en artes, deben lograr explicitar, por una parte, una diferenciación clara entre prácticas artísticas que no tienen por objeto la puesta en marcha de procesos investigativos frente a las que sí lo hacen y, en segundo lugar, frente a estas últimas, explicitar en qué consiste tal apuesta investigativa, cuáles son sus rasgos esenciales y los tipos de saber que constituyen. Uno de esos rasgos de diferenciación es la articulación entre: 1. El objeto, el cual, alude a la composición, la imagen, la actuación el diseño, es decir, lo que comprendemos por obra, 2. El proceso, el cual representa la etapa de producción, y 3, El contexto, que alude al entorno social, cultural, histórico en el que se enmarca el proceso de investigación en artes. De este modo, existe una gran diferencia entre examinar y evaluar resultados o productos en forma de objetos u obras terminadas y evaluar además de ello, la documentación y sistematización del proceso llevado a cabo, así como las relaciones, tensiones o problematización con el contexto en el que tales procesos y objetos se inscriben. Si bien las prácticas artísticas, en sí mismas, contribuyen a reflexionar y problematizar cuestiones insertas en la vida colectiva de las sociedades, podemos hablar de investigación en artes "sólo cuando la práctica artística ofrece una contribución intencionada y original a aquello que ya conocemos y entendemos" (Borgdoff, 2012, p. 15).

Es, justamente, a partir de estos desplazamientos como hemos podido redimensionar lo que estamos en capacidad de nombrar como arte y creatividad y lo que podemos llegar a considera como formas de conocimiento y saber, y es también a partir de estas mutaciones, como podemos reubicar una serie de nociones que han tenido una carga semántica importante en la tradición occidental. Nos referimos a los conceptos de proyecto y obra, y laboratorio y taller.

\section{El proyecto y la obra}

En el ámbito de la investigación, tanto en el campo de las ciencias naturales como de las ciencias sociales y humanas, el "proyecto" representa un constructo en el que está contenido un universo más o menos controlable de preguntas, rutas de indagación, formas de experimentación y marcos comprensivos, frente a un problema, un aspecto relevante, o cuando menos, susceptible de interés para una colectividad particular. Desde su origen etimológico la noción de proyecto contiene, de manera simultánea, la alusión a lo porvenir, en el sentido de "ir hacia delante", pero también la referencia a lo azaroso en el sentido de su indeterminación. No se proyecta un camino seguro, se proyecta una ruta en la que hay indicios, huellas, que hay que seguir, que hay que descifrar. Sin embargo, el proyecto, lleva en su propia condición una suerte de imposibilidad de culminación o de cierre definitivo. Todo punto de llegada es parcial, momentáneo, termina abriendo más el horizonte de indagación, genera más preguntas que certezas y, por ende, impulsa nuevas búsquedas.

La noción de "obra", por su parte, está ligada al trabajo, a la labor, a la relación productiva con la materia. En relación con las prácticas artísticas, la noción de obra, permite el encuentro de la materialidad y la intervención creativa del artista, y en torno a ella tiene lugar la formalización del proceso creativo. Nancy, (2013) señala cómo desde el siglo XIX, la noción de obra se condensa en un sentido particular y privilegiado como "obra de arte", la cual "designa el producto o un conjunto de productos de la actividad de un artista" ( $p$. 73). En el presente, esta noción se expande de un sentido limitado al despliegue y realización de la técnica para configurarse como forma de comprensión del obrar humano, como trabajo sobre la realidad, del cual no se separa su construcción reflexiva. Al exteriorizarse como trabajo artístico, este obrar deviene una acción que excede su naturaleza útil, o por lo menos concreta de finalización de una labor. Es decir, la obra se configura, desde este punto de vista, en un excedente de energía, de sentido, de posibilidad expresiva sobre el mundo y sobre la realidad: "La obra -advierte Nancy- no acaba y este inacabamiento desmiente la seguridad y la completud del objetivo alcanzado" (2013, p. 76).

La tensión configurada entre el constante ir en búsqueda de los indicios y las huellas por medio de un proceso metódico que determina la noción de "proyecto" y la naturaleza inacabada de la acción de "obrar" o de "hacer obra", es lo que nos permite configurar un escenario de interacción para la investigación-creación. De esta manera, una de las expresiones que nombra esta tensión denominada investigación-creación, puede ser la acuñada inicialmente por Joyce y retomada por Nancy de "work in progress", por medio de la cual el trabajo, "el obrar", está siempre empujando el proyecto, mientras que el "proyecto", a su vez, está abriendo de manera constante nuevas preguntas y posibilidades para el "obrar". Trabajo en proceso implica aquí la puesta en límite de esta tensión, cuyas materializaciones sólo alcanzan a ser esbozos, gestos, lugares provisionales de la investigación y la creación

\section{El laboratorio y el taller}

La noción de Laboratorio está ligada etimológicamente a la labor, al trabajo, al uso e implementación de la técnica. La de Taller remite al latín astella, corte o astilla de madera, y encuentra su cercanía en su significación en el castellano con el término francés atalier para hacer referencia al trabajo artesanal o aquella labor realizada fundamentalmente con las manos. De acuerdo con Richard Sennett (2009, p. 73) el taller es el espacio productivo en el que la labor creativa y la efectividad de la vida se entrecruzan; el lugar donde las habilidades se constituyen en fuente de legitimidad del trabajo autónomo. No obstante, la óptica de la tradición moderna irradia sentidos muy concretos para estas dos nociones. El Laboratorio designa el espacio de experimentación, del trabajo práctico en el que 
suceden los procesos de comprobación de la ciencia moderna, la experimentación. el despliegue técnico, la aplicabilidad de las fórmulas, el ensayo y el error. En el contexto contemporáneo este espacio, se propone también como escenario formativo y de transmisión de saberes, en el sentido en el que los aprendices o los investigadores en formación, replican y ponen en prácticas los direccionamientos del investigador titular.

La noción de taller, por su parte, ha estado vinculada al trabajo manual de construcción de herramientas y artesanías, a la labor de transformación de la materia prima y a la aplicación de la técnica. Ya en los inicios de la modernidad, aparece la noción de taller de artista, como espacio reservado para el despliegue de la creatividad. El taller entonces, surge más vinculado al ámbito de la creación, primero, como un espacio de potenciación de la genialidad, en el que el artista se pone en relación con un número limitado de aprendices a quienes instruye en su maestría. Pero también, desde esta connotación, concita un espacio de transmisión de un saberhacer, no basado en fórmulas de estricta aplicación formal, pero sí en técnicas expresivas que han servido de soporte y despliegue a las capacidades creadoras. Por eso, tanto en el Taller como en el Laboratorio la interacción y el trabajo colaborativo resultan ser los medios más eficaces para la labor creativa, dado que en estos escenarios el trabajo se localiza, se moldea, se pone en relación con los otros, animando el diálogo inusitado y la transmisión directa de los saberes.

\section{CONCLUSIONES}

Justamente estas reflexiones fueron el referente para asumir los desafíos que representó abordar la investigación-creación en el contexto de la Maestría en Estética y Creación en la Universidad Tecnológica de Pereira, Colombia; un programa que apuesta por articular los contextos ampliados del arte, la estética y la cultura desde un horizonte multidisciplinar en el que puedan converger formas plurales de concebir la investigación y configurar la creación.

Si bien persisten la necesidad de considerar unos lineamientos claros que orienten la naturaleza, tanto del proceso formativo, como de la construcción de los trabajos de grado de los estudiantes del programa, el interés central del posgrado no está en la formalización de un método o metodología específica. Por el contrario, el programa ha buscado propiciar diferentes vías de acceso para la configuración de proyectos en los que sea posible señalar contextos, reflexionar temas, allanar rutas de aproximación y animar procesos expresivos, en los cuales, se articulen tanto la investigación como la creación de una forma equilibrada y en constante interacción. Es la multiplicidad y no la singularidad la que determina el rasgo epocal de la creación artística contemporáneo. Así sustentó Brea (2004) cuando expresó: “El tiempo en que las artes tenían por misión respaldar el imaginario de un mundo de los seres particulares es un tiempo pasado, muerto. Y felizmente muerto, por negador del ser en su despliegue infinitésimo e innumerable, como epifanía radical de la diferencia". La sucesión entre sistemas de producción y reproducción configura un motor para la cultura, y una estrategia renovadora para deponer lo absoluto, lo estable y lo excluyente como preceptos de la creación artística a favor de lo pluriforme, lo inestable, lo incluyente.

\section{FUENTES REFERENCIALES}

Mandoki, K. (2008). Estética cotidiana y juegos de la cultura. Prosaica I. México: Siglo XXI Ed.

Rancière, J. (2012). El malestar en la estética. Madrid: Clave Intelectual.

Danto, A. (1999). Después del fin del arte. El arte contemporáneo y el linde de la historia. Barcelona: Paidós.

Greenberg, C. (1979). Arte y cultura. Ensayos críticos. Barcelona: Gustavo Gili.

Borgdorff, H. (2012). The conflict of the faculties: perspectives on artistic research and academia. Amsterdam: Leiden University Press.

Nancy, J. L. (2013). La partición de las artes. Valencia: Pre-textos.

Sennett, R. (2009). El artesano. Barcelona: Anagrama.

Brea, Jorge Luis (2004). El fin de la obra singular, en El tercer umbral. Estatuto de las prácticas artísticas en la era del capitalismo cultural. Murcia: CENDEAC. 